\title{
Modeling of photoconversion efficiency for hydrogenated amorphous Si $p$-i-n structures
}

\author{
A.V. Sachenko ${ }^{1 *}$, I.O. Sokolovskyi ${ }^{1}$, A. Kazakevitch ${ }^{2}$, A.I. Shkrebtii ${ }^{2}$, F. Gaspari ${ }^{2}$ \\ ${ }^{I}$ V. Lashkaryov Institute of Semiconductor Physics, NAS of Ukraine, 03028 Kyiv, Ukraine \\ ${ }^{2}$ University of Ontario Institute of Technology, Oshawa, ON, L1H 7L7, Canada \\ *Corresponding author, e-mail: sachenko@inbox.ru
}

\begin{abstract}
An analytical formalism to optimize the photoconversion efficiency $\eta$ of hydrogenated amorphous silicon-based $(\mathrm{a}-\mathrm{Si}: \mathrm{H})$ solar cells has been developed. This model allows firstly the optimization of a $p^{+}-i-n$ sandwich in terms of carrier mobilities, thickness of the layers, doping levels, and others. Second, the geometry of grid fingers that conduct the photocurrent to the bus bars and $\mathrm{ITO} / \mathrm{SiO}_{2}$ layers has been optimized, and the effect of non-zero incidence angles of Sun's light has been included as well. The optimization method has been applied to typical a-Si:H solar cells. The codes allow the optimization of amorphous Si based solar cells in a wide range of parameters and are available on the e-mail request.
\end{abstract}

Keywords: photoconversion efficiency, hydrogenated amorphous silicon, a-Si:H solar cell.

Manuscript received 26.11.07; accepted for publication 19.12.07; published online 31.01.08.

\section{Introduction}

Thin film hydrogenated amorphous silicon (a-Si:H) is widely used for photovoltaic applications [1]. Amorphous silicon-based solar cells (SC) are very promising because of low production cost, possibility of covering large uneven areas, and sufficiently high efficiency. In order to get the best possible performance of the a-Si:H solar cells, it is important to (i) produce high-quality amorphous films with $p-i-n$ junction and (ii) optimize the films and solar cells by their parameters such as, for instance, $p$-, $i$ - and $n$-layer thicknesses, their doping levels, electron and hole mobilities $\mu_{n}$ and $\mu_{p}$, lifetimes of electrons and holes, resistance of $p$-, $i$ - and $n$-layers, contact grid geometry, and parameters of transparent conducting and antireflecting layers.

Many experimental techniques to grow a-Si:H films have been proposed recently. Many of them require a reasonably high substrate temperature to grow high-quality samples. One of the authors (F.G.) has developed the so-called dc saddle-field glow-discharge technique (see [2] and references therein). This efficient technique was used to deposit a hydrogenated thin $\mathrm{Si}$ film on the amorphous solar cells under consideration. It was previously demonstrated that such films can be successfully used for photovoltaic applications [3].

Regarding the theoretical models, a few approaches have been proposed to optimize the performance of solar cells of various types [1]. They range from the analytical models [4] and combined analytical and numerical approaches $[4,5]$ to purely numerical models using a specially developed software such as AMPS. Analytical models have the advantage of being physically intuitive and predictive on a wide range of the cell parameters and offer a possibility of the quick and accurate estimation of photoconversion efficiency including the optimization of the solar cell geometry. However, the common approaches to characterize analytically hydrogenated amorphous silicon solar cells were mainly based on the models developed for a crystalline semiconductor. To extend these models to amorphous $\mathrm{Si}$, the crystalline $\mathrm{Si}$ bandgap $E_{g}$ was simply substituted by the mobility gap $E_{\mu}$ for an amorphous material [6]. In such approaches, the main distinction between amorphous and crystalline Si-based solar cells was due to different resistances of the bases ( $i$-layers). However, to properly characterize a-Si:H solar cells, this is not sufficient. In addition, the Stark effect must be taken into account. For instance, the series resistance of $\mathrm{SC}$ built on $p-i-n$ structures of hydrogenated amorphous silicon is primarily determined by the photoresistance of the $i$-layer (in contrast to crystalline $\mathrm{Si}$ ). It is commonly accepted that this important factor determines the fill factor $\gamma$ of the $I-V$ characteristic of $p-i-n$ structures [1]. An extra parameter responsible for $\gamma$ is $\gamma_{m}$. This is the ratio of the maximum power $P_{M P P}$ at the so-called maximum power point 
(MPP) to the product of the short-circuit current $I_{S C}$ and the open-circuit voltage $V_{O C}$ [2]. Since $P_{M P P}=I_{m} V_{m}\left(I_{m}\right.$ and $V_{m}$ are the current and the voltage at MPP), $\gamma_{m}=$ $I_{m} V_{m} / I_{S C} V_{O C}[2]$. The above theoretical models produce $\gamma_{m}$ for typical hydrogenated amorphous silicon-based SC in a range of $0.81-0.83$, which is always higher than experimental values [1]. This is due to the extra photovoltage drops on the photoresistance of the $i$-layer and the layer resistance of a front contact and a contact net. This has to be included in the analytical model as well. Another problem with the above standard approaches is that they are based on the so-called diode theory of rectification which works well for the crystalline $\mathrm{Si}$ based SC. For a a-Si:H based solar cell, however, the diffusion theory of rectification is more suitable because of the high defect density and the absence of a longrange order, which essentially reduces the mobility of carriers and their lifetime.

Two of the authors (A.V.S. and A.I.S.) have developed analytical two-dimensional models for the optimization of crystalline solar cells [7]. The onedimensional analytical model of photoconversion efficiency for amorphous silicon-based solar cells has been proposed by one of the author (A.V.S.) [8]. Although analytical one-dimensional photoconversion models have been successfully developed in the past $[8,9]$, numerical methods are being mainly used recently to optimize solar cells, especially amorphous Si-based ones [10].

In this paper, we develop an analytical model of photoconversion $\eta$ for hydrogenated amorphous silicon a-Si:H based $p-i-n$ SC under the AM0 condition. The model allows the straightforward extension to other illumination conditions, such as, for instance, AM1.5. The model considers an interplay of various factors such as $p-, i-$, and $n$-layer doping levels and conductivity, carrier mobilities $\mu_{h}$ and $\mu_{p}$, lifetimes of carriers, diffusion length, contact grid geometry, etc. Finally, the solar cells might not be optimally oriented with respect to the Sun, and the additional optimization with respect to the angle of light incidence is included in the model as well. We will demonstrate how the above factors influence the fill factor $\gamma$ of the $I-V$ characteristic. We consider the mobility gap (or electric bandgap) $E_{\mu}$ of amorphous Si to be $1.71 \mathrm{eV}$, although the theory is suitable for various values of this gap. A spectral peculiarity of the photocurrent collection that originates from the diffusion theory of rectification [7] is taken into account as well. Theoretical results were compared with available experimental data and a good agreement has been found.

\section{Theory}

We consider a conventional SC of the sandwich type consisting of the $p-i-n$ structure between the frontal grid and rear electrodes (see Fig. 1). The frontal collecting grid electrode consists of parallel metal fingers connected to one another through two conductive bus bars. The grid is placed on the top of a conductive $p$-type ITO film of thickness $d_{2}$. The area between fingers is filled with an antireflecting $\mathrm{SiO}_{2}$ film with thickness of $d_{1}$. The highly doped $p$-layer of thickness $d_{p}$ is in contact with the frontal electrode on the top and the $i$-layer of thickness $d$ on the bottom. The highly doped $n$-layer of thickness $d_{n}$ is located between the $i$-layer and the uniform $\mathrm{Al}$ rear electrode with reflectivity $R_{d}$ (Fig. 1).

The frontal grid and rear electrodes collect photocurrent. ITO film (i) serves as a wideband window; (ii) provides the transparency for a solar illumination; and (iii) collects the photocurrent between metal fingers of the grid electrode. The highly doped $p$ and $n$ layers create a rectifying barrier that separate electrons and holes, while the electron-hole pair generation takes place mainly in the $i$-layer.

In the case of the single reflection from the metal rear electrode, the electron-hole pair generation function $g(\alpha, x)$ at a given wavelength $\lambda$ is described by

$$
\begin{aligned}
& g(\alpha, x)=\alpha \cdot I \cdot[\exp (-\alpha x)+ \\
& \left.+R_{d} \cdot \exp \left(-2 \alpha\left(d+d_{p}+d_{n}\right)+\alpha x\right)\right]
\end{aligned}
$$

where $\alpha=\alpha(\lambda)$ is the wavelength-dependent absorption coefficient, and $I$ is the intensity of monochromatic light at the wavelength $\lambda$.

The $\alpha(\lambda)$ dependence for hydrogenated amorphous silicon is taken from our experimental measurements (previously, the earlier results [8] were used). Since the absorption coefficient essentially depends on the photon energy, we distinguish (i) the high-energy part of the spectrum (above $E_{\mu}$ ), (ii) intermediate energy (near $E_{\mu}$ ), and (iii) the energy below the mobility gap $E_{\mu}$, in the socalled exponential bandtail. In the present paper, we take $E_{\mu}=1.71 \mathrm{eV}$.

The analytical procedure of calculation of the hole diffusion flux at the $n^{+}$-layer boundary and that of electrons in the $i$-layer in the plane $x=x_{n}$ is outlined in [5]. This gives the electron collection coefficient in the $p$-layer $f_{n}(\alpha)$ as follows:

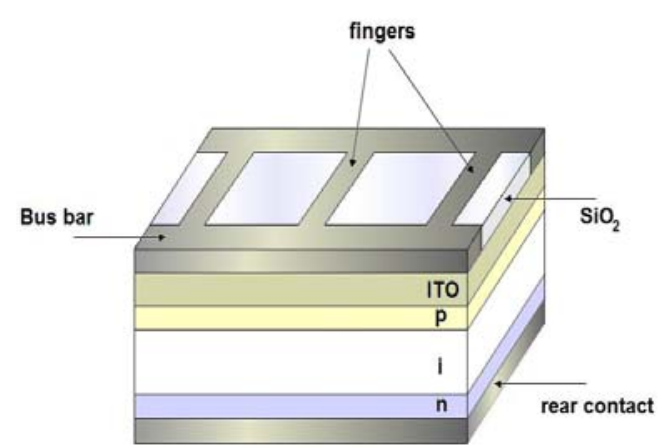

Fig. 1. Schematic view of a solar cell under consideration. It consists of the $p-i-n$ structure between frontal grids and the rear contact. The frontal collecting grid electrode contains parallel metal fingers connected to one another through two conductive bus bars. The frontal grid is placed on the top of the transparent conducting ITO layer. 


$$
\begin{aligned}
& f_{n}(\alpha)=\frac{\alpha L_{n}}{(\alpha L n)^{2}-1} \times\left[S_{0} \sinh \left(\frac{d_{p}}{L_{n}}\right)+V_{p} \cosh \left(\frac{d_{p}}{L_{n}}\right)\right]^{-1} \times \\
& \times\left\{\alpha D_{n}+S_{0}\left(1-\exp \left(-\alpha d_{p}\right) \cosh \left(\frac{d_{p}}{L n}\right)\right) \times\right. \\
& -V_{n} \exp \left(-\alpha d_{p}\right) \sinh \left(\frac{d_{p}}{L n}\right)-\alpha L_{n} \exp \left(-\alpha d_{p}\right) \times \\
& \left.\left[S_{0} \sinh \left(\frac{d_{p}}{L_{n}}\right)+V_{n} \cosh \left(\frac{d_{p}}{L_{n}}\right)\right]\right\} .
\end{aligned}
$$

The hole collection coefficient in the $i$-layer is

$$
\begin{aligned}
& f_{p}(\alpha) \cong-\frac{\alpha L}{\left(\alpha^{2} L^{2}-1\right)} \cdot\left\{\left[\left(S_{d}+V\right) \cdot \exp \left(\frac{d}{L}\right)+\right.\right. \\
& \left.+\left(S_{d}-V\right) \cdot \exp \left(\frac{-d}{L}\right)\right] \times \\
& \times\left[\exp \left(-\alpha d_{p}\right)+R_{d} \exp \left(-2 \alpha d-\alpha d_{p}\right)\right]+ \\
& \left.+2\left[\alpha D\left(1-R_{d}\right)-S_{d}\left(1+R_{d}\right)\right] \exp \left(-\alpha\left(d+d_{p}\right)\right)\right\} \times \\
& \times\left[\left(S_{d}+V\right) \cdot \exp \left(\frac{d}{L}\right)-\left(S_{d}-V\right) \cdot \exp \left(\frac{-d}{L}\right)\right]^{-1}+ \\
& +\frac{(\alpha L)^{2}\left[\exp \left(-\alpha d_{p}\right)-R_{d} \exp \left(-2 \alpha d-\alpha d_{p}\right)\right]}{\left(\alpha^{2} L^{2}-1\right)} .
\end{aligned}
$$

Here, $L_{n}=\sqrt{D_{n} \tau_{n}}, V_{n}=D_{n} / L_{n}, D_{n}$ is the electron diffusion coefficient in the $p^{+}$-layer, $L=\sqrt{D \tau_{p}}$, $V=D / L, D$ is the diffusion coefficient of holes in the $i$ layer, $S_{0}$ and $S_{d}$ are surface recombination velocities at the illuminated (top) and rear surfaces, respectively.

As was mentioned above, the diffusion theory of rectification has to be used for hydrogenated amorphous silicon, and the total electron-hole pair collection coefficient $f(\alpha)$ is $[7,9]$

$$
f(\alpha) \cong f_{n}(\alpha)+f_{p}(\alpha)-\frac{\alpha L_{s}}{1+\alpha L_{s}} \exp \left(-\alpha d_{p}\right) .
$$

Here, $L_{S}=k T / q E_{m}$, and $E_{m}$ is the maximum electrical field in the $p^{+}-i$ junction.

The last term in (4) is responsible for a decrease of the collection coefficient at short wavelengths. This happens even if the surface recombination velocity $S_{0}$ and the bulk recombination in the $p$-layer can be neglected. The calculated spectral dependence of the collection coefficients for this case is shown in Fig. 2. It is clear from the plot that the stronger the maximum value of the electric field $E_{m}$, the higher is the maximum of the collection coefficient $f$. The increase of $E_{m}$ shifts the region where the collection coefficient decreases toward shorter wavelengths.

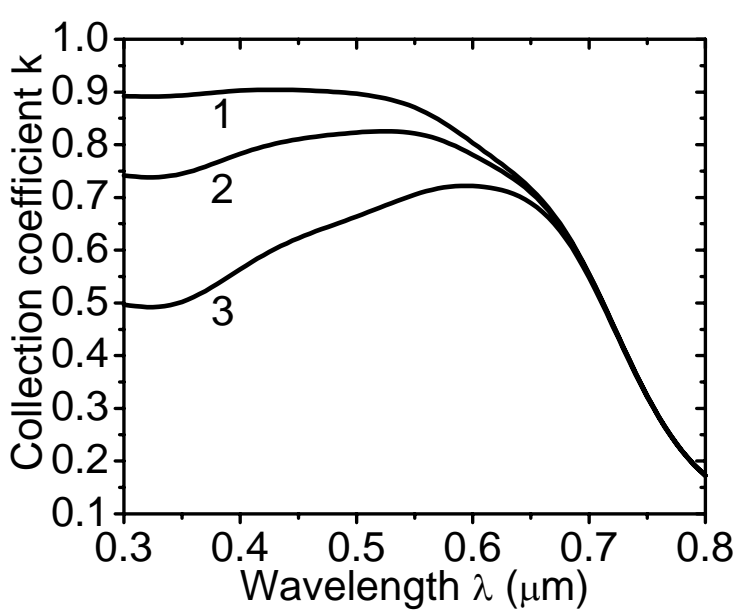

Fig. 2. Spectral dependences of the collection coefficient in a-Si:H-based SC for different values of the maximum electric field $E_{m}$ equal to $10^{5}, 3 \cdot 10^{4}$, and $10^{4} \mathrm{~V} / \mathrm{m}$ : curves 1,2 , and 3 , respectively.

Equation (5) gives the short-circuit current $J_{S C}$ for the AM0 conditions. Approximating AM0 by the black body radiation at $T_{c}=5800 \mathrm{~K}$, taking the mobility gap to be $1.71 \mathrm{eV}$, measuring the $J_{S C}$ current density in $\mathrm{A} / \mathrm{cm}^{2}$, and combining the fundamental constants, we obtain a dimensionless convenient expression for the short-circuit current $J_{S C}$ in the form

$$
\begin{aligned}
& J_{S C}(\varphi)=\frac{2 c}{\lambda_{x}^{3}}\left(\frac{r_{S}}{D_{S}}\right)^{2} \pi q \cdot(1-m) \times \\
& \times \int_{0}^{1} \frac{f(\alpha(z)) \cdot\left(1-R_{S}(z, \varphi)\right) \cdot \cos \varphi}{z^{4} \cdot\left[\exp \left(\frac{h c}{\lambda_{x} k T \cdot z}\right)-1\right]} d z .
\end{aligned}
$$

Here, $\varphi$ is the sunlight incidence angle, $m$ is the frontal grid shadowing coefficient $(m \leq 1$, the bigger $m$ is, the smaller part of the solar cell is illuminated), $R_{S}(z, \varphi)$ is the reflection coefficient of the SC frontal surface, $z=\lambda / \lambda_{x}, \lambda$ is the wavelength of the incident light, $\lambda_{x}$ is the threshold wavelength of the photoelectric effect for hydrogenated amorphous silicon, $r_{S}$ is the equatorial radius of the Sun, and $D_{S}$ is the mean SunEarth distance.

We used $\lambda_{x}=0.8 \mu \mathrm{m}$, which corresponds to the absorption edge of an a-Si:H film for $E_{\mu}=1.71 \mathrm{eV}$.

The spectral dependence of the reflection coefficient $R_{S}(z, \varphi)$ for a multilayer structure $\mathrm{SiO}_{2}-$ ITO $-\alpha-\mathrm{Si}: \mathrm{H}$ was calculated according to Berning's approach [6] which considers the oblique incidence of the sunlight at an angle $\varphi$. To maximize the short-circuit current density $J_{S C}$, the reflections from the SC should be as low as possible. For the typical refractive index of $\mathrm{SiO}_{2}(n=1.45)$ and ITO $(n=1.96)$, the minimal reflection of the $\mathrm{SiO}_{2}$-ITO structure occurs when the $\mathrm{SiO}_{2}$ film is $0.09 \mu \mathrm{m}$ thick, and the ITO layer is $1.5 \mu \mathrm{m}$ thick. 
The angular dependence of the short circuit current density $J_{S C}$ normalized to its maximum value is shown in Fig. 3. The angle $\varphi$ corresponds to Earth's axis inclination with respect to the normal to the ecliptic plane. The short-circuit current density at $\varphi \approx 23^{\circ}$ is $91 \%$ of that at $\varphi=0$, while $J_{S C}$ is $93 \%$ of its maximum value for the angle-independent reflection coefficient $R_{S}$.

It is instructive to consider the upper limit of the

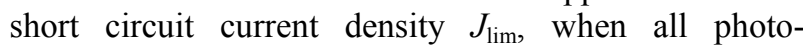
generated carriers in the $i$-layer are collected:

$$
\begin{aligned}
& J_{\lim } \cong \frac{2 c}{\lambda_{x}^{3}}\left(\frac{r_{S}}{D}\right)^{2} \pi q \cdot(1-m) q \times \\
& \times \int_{0}^{1} \frac{\left[1-\exp \left(-\alpha d+R_{d} \exp (-2 \alpha d)(\exp (\alpha d)-1)\right] \cdot\left(1-R_{S}(z)\right)\right.}{z^{4} \cdot\left[\exp \left(\frac{h c}{\lambda_{x} k T \cdot z}\right)-1\right]} d z .
\end{aligned}
$$

Figure 4 demonstrates $J_{\lim }$ and $J_{S C}$ dependence when the $i$-layer thickness $d$ changes from 0.1 to $10 \mu \mathrm{m}$. As seen, $J_{\text {lim }}$ monotonically increases, as $d$ grows (curve 1). When the rear contact is not reflective $\left(R_{d}=0\right)$, the density of the short-circuit current $J_{S C}$ increases with $d$ and saturates after $1 \mu \mathrm{m}$ (curve 6). Curves from 2 to 5 located between $J_{\lim }(d)$ at $R_{d}=1$ and $J_{S C}(d)$ at $R_{d}=0$ correspond to $R_{d}$ values of $0.8,0.6,0.4$, and 0.2 . The current density reaches its maximum when $d_{m} \approx L$ and increases, as the bulk recombination velocity $S_{d}$ decreases. Finally, $J_{S C}(d)$ approaches $J_{\lim }(d)$ when the diffusion length $L$ is much larger than $d$, and the surface recombination $S_{0}$ as well as $S_{d}$ can be neglected.

The standard expression for the photocurrent $J_{\mathrm{ph}}$, (or solar cell $I-V$ characteristic) in terms of the diode ideality factor $A$ and the saturated current density $J_{S}$ is as follows:

$$
J_{\mathrm{ph}}(V)=J_{S C}-J_{S} \exp \left(\frac{q V}{A k T}\right) .
$$

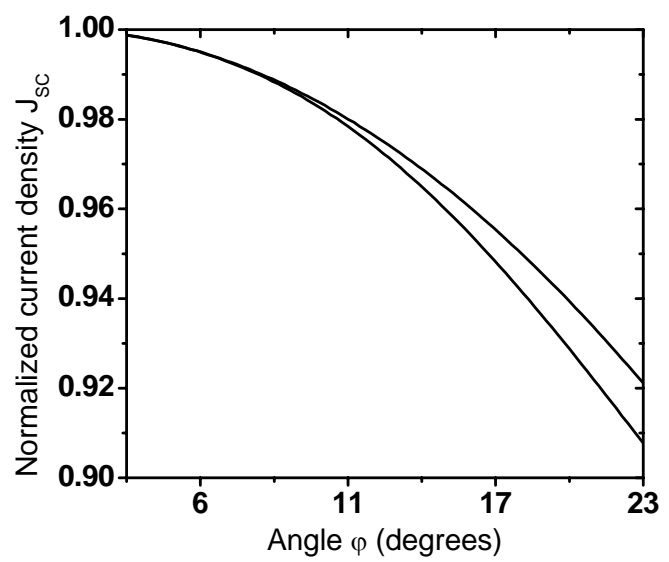

Fig. 3. Normalized angular dependence of the short-current density $J_{S C}$ for a-Si:H-based SC. Curve 1 includes the reflection coefficient dependence on the light incidence angle $\varphi$, while curve 2 does not consider it.

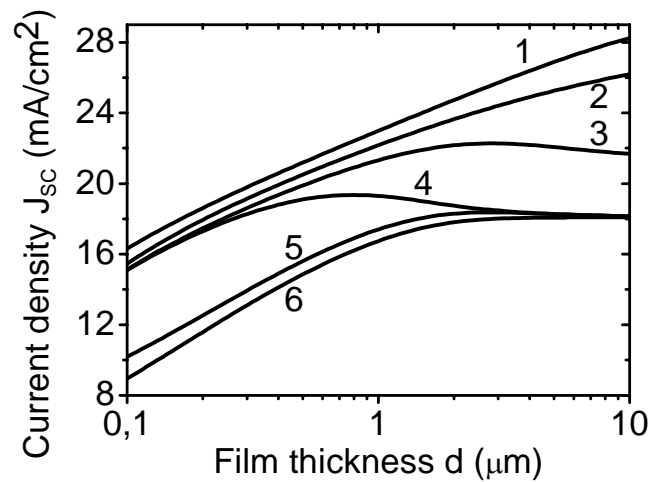

Fig. 4. Photocurrent density versus the $i$-layer thickness $d$. Curve 1 corresponds to the maximally possible photocurrent density $J_{\text {lim }}(d)$, when all the light is adsorbed in the film. Curves 2-6 correspond to the photocurrent $J_{S C}(d)$. For curve 2, the recombination is minimal, and the field is maximal. For curve $6, R_{d}=0$. For curve $3, t=10^{-5} \mathrm{~s}$, for curves 4,5 , and 6 , $t=10^{-6} \mathrm{~s}$. For curves 5 and $6, S_{0}=10^{2} \mathrm{~cm} / \mathrm{s}, S_{d}=10^{4} \mathrm{~cm} / \mathrm{s}$. For curves $3,4, S_{0}=10^{2} \mathrm{~cm} / \mathrm{s}, S_{d}=10 \mathrm{~cm} / \mathrm{s}$.

The open-circuit voltage $V_{O C}$ of the SC obtained from (6) by putting $J_{\mathrm{ph}}=0$ is

$V_{O C}=\frac{A k T}{q} \ln \left(\frac{J_{S C}}{J_{S}}\right)$.

The expression for the photoconversion efficiency under the AM0 condition $\eta_{\mathrm{AM} 0}$ can be written [2] as

$$
\begin{aligned}
& \eta_{A M 0} \cong \frac{S J_{S C} V_{O C}}{P}\left(1-\frac{1}{q V_{O C} / A k T}\right) \\
& \times\left(1-\frac{\ln \left(q V_{O C} / A k T\right)}{q V_{O C} / A k T}\right) \times\left(1-\frac{S J_{S C}\left(R_{S C}+R_{\mathrm{ph}}\right)}{V_{O C}}\right),
\end{aligned}
$$

where $S$ is the SC area, $P=0.135 \mathrm{~W} / \mathrm{cm}^{2}$ is the power of incident solar radiation under the AM0 condition, $R_{S C}$ is the component of the series layer resistance of the ITO film and the grid electrode, $R_{\mathrm{ph}}$ is the component of the series resistance of photogenerated carriers in the $i$-layer.

If $S J_{S C} R_{S C} / V_{O C}<<1$ and $S J_{S C} R_{\mathrm{ph}} / V_{O C}<<1$, the last term of Eq. (8) can be rewritten as $\gamma_{R} \gamma_{\sigma}$, where $\gamma_{R}$ and $\gamma_{\sigma}$ are multipliers of the fill factor responsible for the resistance of the grid electrode and the $i$-layer, respectively. This product demonstrates the influence of the series resistance of $\mathrm{SC}$ on the fill factor of the $I-V$ characteristic. The value of $\gamma_{R}$ determines the influence of the $\mathrm{SiO}_{2}$ layer resistance and the grid electrode resistance; and $\gamma_{\sigma}$ is related to the $i$-layer photoresistance.

While determining the series resistance $R_{\mathrm{ph}}$ of the $i$ region, we consider the electron mobility much over the hole mobility. Then

$$
R_{\mathrm{ph}}=\frac{1}{S q \mu_{n}} \int_{0}^{d} \frac{d x}{n_{0}+\Delta n(x)},
$$


where $S$ is the sample surface area, $\mu_{n}$ the electron mobility, $n_{0}$ the equilibrium electron concentration in the $i$-region, and $\Delta n(x)$ the increase of the electron concentration under illumination. The $\Delta n(x)$ value is determined from the relation

$$
\begin{aligned}
& \Delta n(x)=\Delta p(x)=C_{1} \exp \left(-\frac{x}{L}\right)+C_{2} \exp \left(\frac{x}{L}\right)+ \\
& +\frac{\alpha I^{*} L^{2}\left(\exp (-\alpha x)+R_{d} \exp (-2 \alpha d+\alpha x)\right)}{D\left(1-\alpha^{2} L^{2}\right)},
\end{aligned}
$$

where $\Delta p(x)$ is the solution of a differential equation for holes in the $i$-region for an open circuit. For AM0 conditions, the $I^{*}$ value is determined from the following formula:

$$
I^{*}(\varphi)=\frac{1.28}{q} \cdot(1-m) \cdot \int_{0}^{1} \frac{\left(1-R_{S}(z, \varphi)\right) \cdot \cos \varphi}{z^{4} \cdot\left[\exp \left(\frac{3.12}{z}\right)-1\right]} d z
$$

After the determination of the integration constants $C_{1}$ and $C_{2}$ from the boundary conditions for the hole flows at $x=d_{p}$ and $x=d_{p}+d$,

$$
\begin{aligned}
& j\left(x=d_{p}\right)=-S_{0} \Delta p\left(x=d_{p}\right), \\
& j\left(x=d_{p}+d\right)=-S_{d} \Delta p\left(x=d_{p}+d\right),
\end{aligned}
$$

and the substitution of the values obtained in Eqs. (10) and (9), one can calculate the component of the $I-V$ curve filling factor $\left(1-S J_{S C} R_{\mathrm{ph}} / V_{O C}\right)$ that is related to the series resistance of the $i$-region.

As the next step, we consider the frontal grid consisting of parallel metal fingers connected to bus bars (see Fig. 1). To determine the grid parameters, we can neglect the resistance of the metal grid electrodes compared to the essentially higher layer resistance of the ITO film. If the inequality $S J_{S C} R_{S C} / V_{O C}<<1$ is satisfied, $\gamma_{R}$ can be written as [11-13]

$$
\gamma_{R}=\frac{2 L_{c}}{l} \tanh \left(\frac{l}{2 L_{c}}\right) \text {. }
$$

Here, $L_{c}=\left[q \mu p N_{p} V_{O C} / J_{O C}\right]^{1 / 2}$ is the effective collection length, $\mu_{p}$ is the hole mobility, $N_{p}=n_{p} d$, and $n_{p}$ is the two-dimensional hole concentration (Gibbs excess) in the $\mathrm{SiO}_{2}$ film.

Equation (11) is accurate when $L_{c}>>l$, where $l$ is the distance between metal fingers. If a metal finger has width $l_{n}$, and $m$ is the relative front-side metallization, that is the shadowing of the photoactive area by the metal grid $(0<m<1)$, the distance between fingers $l \cong l_{n}(1-m) / m$ (see [9]).

Since the open-circuit voltage $V_{O C}$ for AM0 is very close to that at AM1.5, the efficiency $\eta_{A M 1.5}$ of SC at AM1.5 can be expressed in terms of the efficiency $\eta_{A M 0}$ at AM0 as

$$
\eta_{A M 1.5} \approx \eta_{A M 0} \cdot \frac{0.135}{0.1} \cdot \frac{J_{S C}^{A M 1.5}}{J_{S C}^{A M 0}} .
$$

To find the peak values of $J_{S C}^{A M 1.5}$, we carried out the numerical integration of Eq. (12) at AM1.5, considering for simplicity that the collection coefficient equals 1 . For $E_{\mu}=1.71 \mathrm{eV}$, the value of $\eta_{A M 1.5}$ is about $10 \%$ higher than that of $\eta_{A M 0}$.

\section{Optimization of parameters of hydrogenated amorphous silicon solar cells}

Using the expressions derived above, we can finally maximize the SC performance. The parameters of SC that have to be optimized include the thicknesses and doping levels of the base ( $i$-layer) and the emitter ( $p$ layer), mobility gap $E_{\mu}$, front-side metallization, emitter layer resistance, loss of power due to the reflection, etc.

Let us carry out the optimization of the $i$-layer thickness taking into account that the efficiency is limited by light absorption for a thin film and by its resistance in the case of thick films. In Fig. 5, the $\eta$ values versus the $i$-layer thickness are displayed. These curves were evaluated for different lifetimes in the $i$ layer, by using (8) and taking into account (9) and (10). The formation of a maximum on curves 1-6 is caused by the existing maxima of $J_{S C}(d)$ dependences at high values of the coefficient of reflection from the rear electrode. Another mechanism deals with the fact that the $i$-layer thickness expansion provides the escalation of the amount of generated pairs due to an increase in the absorption. But, on the other hand, extending the base layer enlarges the series resistance related to the $i$-layer photoresistance that produces the photovoltage drop. At low values of either the surface recombination velocity at the rear surface $S_{d}$ or the photoelectron lifetime in the base, the maximum of $\eta$ shifts to smaller values of emitter thickness, $d$; while larger $S_{d}$ values cause the maximum to move to larger $d$. The calculated values of $d_{m}$ vary from $3 \cdot 10^{-5}$ to $10^{-4} \mathrm{~cm}$, which correlates with experimental data.

As is well known, the Staebler-Wronski effect leads to a sufficient escalation of the density of states that causes a comparative reduction of the electron and hole lifetimes in the $i$-layer. Therefore, curves 1 and 2 can be interpreted as the efficiency of photoconversion in SC based on hydrogenated amorphous silicon according to the Staebler-Wronski effect. In this case, the efficiency maximum value reaches $\sim 8 \%$ that is comparable with experimental data for high-efficient SC.

Thus, from the data obtained, we can conclude that the thickness of the base ( $i$-layer) is required to be optimized first as a key parameter.

The following parameters were used in plotting the curves in Fig. 5: $D=2.5 \cdot 10^{-3} \mathrm{~cm}^{2} / \mathrm{s}, \quad R_{d}=0.9$; $E=10^{5} \mathrm{~V} / \mathrm{cm} ; \quad R_{S}=0.082 ; \quad J_{S}=10^{-12} \mathrm{~A} / \mathrm{cm}^{2} ; \quad A=1.5 ;$ $T=300 \mathrm{~K} ; \mu_{n}=0.1 \mathrm{~cm}^{2} /(\mathrm{V} \cdot \mathrm{s}) ; \mu_{p}=10^{2} \mathrm{~cm}^{2} /(\mathrm{V} \cdot \mathrm{s}) ; N=$ $10^{15} \mathrm{~cm}^{-2} ; m=0.05$. The values $J_{S}=10^{-12} \mathrm{~A} / \mathrm{cm}^{2}$ and $A=1.5$ are taken from [1] and correspond to the parameters of high-efficient SC. 


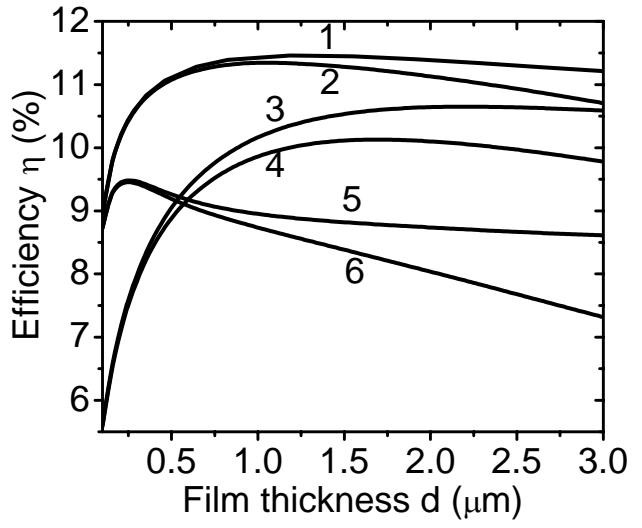

Fig. 5. Efficiency as a function of the $i$-layer thickness. For curves 1,3 , and $5, n_{0}=10^{15} \mathrm{~cm}^{-3}$; for curves 2,4 , and $6, n_{0}=$ $10^{14} \mathrm{~cm}^{-3}$. For curves 3 and $4, S_{0}=10^{5} \mathrm{~cm} / \mathrm{s}, S_{d}=10^{4} \mathrm{~cm} / \mathrm{s}$. For curves $1,2,5$, and $6, S_{0}=S_{d}=10^{2} \mathrm{~cm} / \mathrm{s}$. For curves 5 and $6, \tau=$ $10^{-7} \mathrm{~s}$, and, for curves $1,2,3$, and $4, \tau=10^{-6} \mathrm{~s}$.

The used values of $\mu_{n}$ and $\tau_{n}$ lie within the corresponding ranges realizable in amorphous hydrogenated silicon (though they should be specified), while the values $\mu_{p}=10^{2} \mathrm{~cm}^{2} /(\mathrm{V} \cdot \mathrm{s})$ and $N=10^{15} \mathrm{~cm}^{-2}$ (belonging to the ITO parameters) were taken arbitrarily. Thus, the typical ITO $p$-layer parameters (hole mobility and concentration) are to be found in the literature.

It was stated in [1, p. 387] that the doped contact $p$ and $n$-layers in SC made of hydrogenated silicon are 5 $10 \mathrm{~nm}$ thick. According to the more accurate data given in the report by B.A. Korevaar (Eindhoven Technical University), the thickness of a $p$-(n-)layer is $10(20) \mathrm{nm}$. In our calculations, we took $d_{p}=10 \mathrm{~nm}$. Strictly speaking, one should consider (taking into account the single reflection of light from the metallized back surface) that light passes a distance $2\left(d+d_{p}+d_{n}\right)$, where $d_{n}$ is the heavily doped $n$-layer thickness. In the above equations, the quantity $d_{n}$ was not allowed for. This led to a change of the reflection coefficients in the $p$ - and $n$ layers, since the mobility gap values in them differ from that in the $i$-region. However, if the conditions $d>>d_{p}, d_{n}$ and $\alpha d_{p} \leq 1$ are satisfied, the corrections due to the allowance for the distinction in $\alpha$ values are sufficiently small. Here, we assumed, when taking the absorption in the $p$-layer into account, that the coefficient of light absorption in it is the same as that in the $i$-region.

Now let us consider the optimization of the contact grid parameters. In this case, the optimization is made with allowance for the fact that, at a small shadowing (i.e., at a big distance between the fingers), the series resistance increases, while the great shadowing (i.e., at a small distance between the fingers) leads to a decrease in the photocurrent.

In Fig. 6, we show the photoconversion efficiency vs the relative degree of metallization, the finger width $l_{n}$ serving as a parameter. When plotting the curves in Fig. 6, we considered that the product of the hole

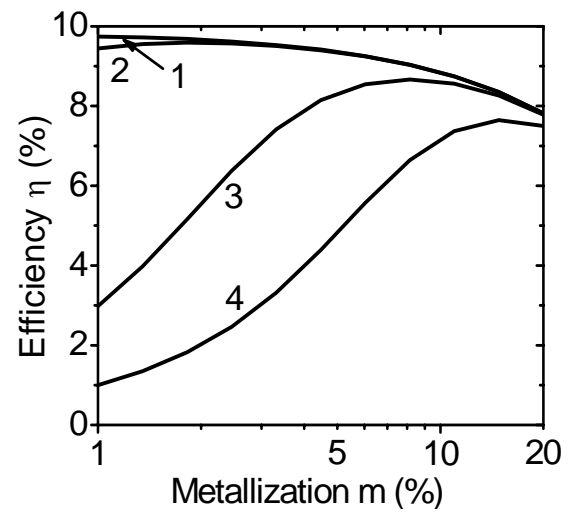

Fig. 6. Efficiency as a function of metallization. $L=3 \cdot 10^{-3}$, $10^{-2}, 10^{-1}$, and $3 \cdot 10^{-1} \mathrm{~cm}$ for curves $1,2,3$, and 4 , respectively.

concentration in the $p$-layer and the layer thickness is $10^{15}\left(10^{14}\right) \mathrm{cm}^{-2}$. An important parameter in this case is also the hole concentration (while plotting the curves in Figs. 5 and 6 , we took it to be $\left.10^{2} \mathrm{~cm}^{2} /(\mathrm{V} \cdot \mathrm{s})\right)$. One can see from the above figures that the bigger the finger width, the higher is the metallization degree, at which the maximal photoconversion efficiency is realized and the lower is the peak photoconversion efficiency. As the values of $\mu_{p}$ and $N$ decrease, one should choose smaller values of the finger width.

\section{Conclusions}

The primary need to optimize the base region ( $i$-layer) thickness can be concluded from the obtained data.

Efficiency as a function of metallization has a maximum.

The efficiency maximum value reaches $\sim 8 \%$ that is comparable with experimental data for high-efficient $\mathrm{SC}$.

\section{References}

1. R.A. Street, Hydrogenated Amorphous Silicon. Cambridge University Press, Cambridge, 1991.

2. A.L. Fahrenbruch, R.H. Bube, Fundamentals of Solar Cells. Photovoltaic Solar Energy Conversion. Academic Press, New York, 1983.

3. Proc. 19th European Photovoltaic Solar Energy Conference, 7-11 June 2004, Paris, France, p. $1374-1377$.

4. E.A. Schiff // Solar Energy Materials \& Solar Cells 78, p. 567-595 (2003).

5. E.A. Schiff // Journal of Non-Crystalline Solids 352(9), p. 1087-1092 (2006).

6. T. Searle (Ed.), Properties of amorphous silicon and its alloys, INSPEC. The Institute of Electrical Engineers, London, 1998.

\section{(C) 2007, V. Lashkaryov Institute of Semiconductor Physics, National Academy of Sciences of Ukraine}


7. A.V. Sachenko // Soviet Phys. Semicond. 19, p. 903 (1985).

8. L. Jiao, I. Chen, R.W. Collins, C.R. Wronski, and N. Hata // Appl. Phys. Lett. 72(9), p. 1057-1059 (1998).

9. A.P. Gorban, V. P. Kostylyov, A.V. Sachenko // Semiconductor Physics, Quantum Electronics \& Optoelectronics 2(3), p. 26-31 (1999).

10. P.H. Berning, in: G. Hass (Ed.), Physics of Thin Films, Vol. 1. Academic Press, New York, 1963, p. $69-121$.
11. A.V. Sachenko, A. I. Chkrebtii // Ukr. Fizicheskiy Zhurnal 29(12), p. 1855-1859 (1984) (in Russian).

12. A.V. Sachenko, A.I. Chkrebtii, T.V. Panichevskaya // Optoelektronika i poluprovodnikovaya tekhnika 21, p. 63-70 (1991) (in Ukrainian).

13. A.V. Sachenko, A.P. Gorban // Semiconductor Physics, Quantum Electronics \& Optoelectronics 2(2), p. 42-44 (1999) (http://www.journalspqeo.org.ua/n2_99/42_299.htm). 